
\title{
Physical Medicine
}

\section{The Effect of Non-Impact Lower Body Exercise on Individuals with Parkinson's Disease: A Case Study}

\author{
Bauer $\mathrm{J}^{1 *}$, Vincent $\mathrm{I}^{2}$, Buckenmeyer $\mathrm{P}^{1}$, Generali $\mathrm{A}^{1}$, and Lind $\mathrm{E}^{1}$ \\ ${ }^{1}$ SUNY Cortland Kinesiology Department, Cortland, NY 13045 USA \\ ${ }^{2}$ SUNY Cortland Communication Disorders \& Sciences
}

\begin{abstract}
Parkinson's disease (PD) is a progressive neurodegenerative syndrome characterized by motor dysfunctions resulting in general physical diminishment that comes from the body's inability to synthesize and utilize dopamine to control muscle action. A 46-year-old mother of two had been experiencing motor and sensory changes for two years and was diagnosed with PD two months prior to volunteering as a participant. The present study reports the effects of exercise training utilizing a Quadmill ${ }^{\mathrm{TM}}$ exercise machine over a 17-week training protocol. The Quadmill ${ }^{\mathrm{TM}}$ eccentrically works lower extremity muscles through repetitive weight-bearing non-impact lower body movement. Biomechanical, physiological, and speech variables often compromised by PD were tracked over the course of the study. Positive acute and chronic improvements were observed in numerous gait and static balance-related variables. Heart rate (bpm), systolic blood pressure $(\mathrm{mmHg})$, and mean arterial pressure (mmHg) increased during the performance of the exercise. Rating of perceived exertion (RPE) was significantly lower with the higher workloads (Phase 2) compared to the lower workloads (Phase 1) during the 17 weeks of exercise sessions. No significant differences in any measured speech-related variables were observed. Results suggest that non-impact lower body exercise using the Quadmill ${ }^{\mathrm{TM}}$ may be beneficial in reducing or reversing gait and balance deficits exhibited by individuals with PD, however, further investigation is needed.
\end{abstract}

Keywords

Non-impact lower body exercise, Parkinson's disease, Quadmill ${ }^{\mathrm{TM}}$, Mobility Lab ${ }^{\mathrm{TM}}$

\section{Introduction}

Parkinson's Disease (PD) is progressive neurodegenerative syndrome characterized by motor dysfunctions such as tremor of the hand/forearm, shuffling gait, general slowing of movement, rigidity, inability to change facial expressions and postural instability [1,2]. It is a condition of physical diminishment resulting from the body's inability to synthesize and utilize dopamine to control muscle action [3]. The disease typically is diagnosed in adults $60+$ years in age; however, Juvenile Parkinson's disease has been identified in individuals as young as six years old [4-6].

Arriving at a diagnosis of PD is often difficult since there are no reliable biomarkers or universally accepted diagnostic tests to identify PD [7]. A diagnosis of PD is commonly made if a patient exhibits tremor, rigidity, bradykinesia and often postural instability in conjunction with the exclusion of other possible causes of Parkinsonism [8]. While there are many symptoms that are common to people suffering from $\mathrm{PD}$, their presentations and severity follow a uniquely indi- vidualized timeline for many. One commonality, however, is the fact that eventually gait, and balance will be affected, reducing a person's mobility and increasing their likelihood of falls [9]. Another frequent consequence of PD is linked to the increased whole-body rigidity experienced as the disease progresses. With the loss of flexibility of the diaphragm and vocal folds it becomes more difficult for individuals to maintain normal speaking volume, pitch and tone. The softer monotone speech patterns that tend to develop in PD sufferers make verbal communication more and more of a challenge $[10,11]$. Speech therapy programs such as the Lee Silverman Voice Technique have been used for years with

\footnotetext{
*Corresponding author: Bauer J, SUNY Cortland Kinesiology Department, Cortland, NY 13045, USA
}

Accepted: September 19, 2018; Published online: September 21, 2018

Citation: Bauer J, Vincent I, Buckenmeyer P, et al. (2018) The Effect of Non-Impact Lower Body Exercise on Individuals with Parkinson's Disease: A Case Study. J Phys Med 1(1):24-31

Copyright: (c) 2018 Bauer J, et al. This is an open-access article distributed under the terms of the Creative Commons Attribution License, which permits unrestricted use, distribution, and reproduction in any medium, provided the original author and source are credited. 
Citation: Bauer J, Vincent I, Buckenmeyer P, et al. (2018) The Effect of Non-Impact Lower Body Exercise on Individuals with Parkinson's Disease: A Case Study. J Phys Med 1(1):24-31

varying levels of success as a method for PD patients to reduce the effects of this common symptom [12].

Finding a safe yet effective mode of exercise for PD patients can be challenging given their wide range of motor and neurological issues. A Quadmill ${ }^{\mathrm{TM}}$ exercise machine provides an exercise movement modality that is time efficient and conducive to ensuring the safety of PD patients. This unique exercise experience consists of repeated body weight squats resulting from its user standing with both feet on a solid platform that follows a prescribed elliptical path. This configuration allows anyone strong enough to stand and support their body weight to use the machine, and since there is no moving tread requiring repositioning of the feet as on a treadmill, there is limited possibility of tripping or injury during use. The non-impact exercise motion of the Quadmill ${ }^{\mathrm{TM}}$ results in the user experiencing flexion/extension at the hip and knee joints and dorsi/plantar flexion at the ankle during each platform revolution. When used correctly, the upper body stays stationary and major muscles of the lower body are repeatedly activated, often causing noticeable quadriceps fatigue even in healthy fit individuals after less than one minute of use.

The purpose of this study was to observe the effects of Quadmill ${ }^{\mathrm{TM}}$ training on symptoms associated with PD over a 17 -week training protocol.

\section{Materials and Methods}

The study was approved by the State University of New York College at Cortland Institutional Review Board, and written informed consent was obtained from the individual prior to participation. Our trial participant volunteered and was identified through the Michael J. Fox Foundation for Parkinson's Research online clinical trial matching tool (https://foxtrialfinder.michaeljfox.org) for patients interested in participating in clinical research.

\section{Case presentation}

The participant was a 46-year-old female, mother of two, who had begun experiencing motor and sensory changes approximately two years prior to involvement in the study. She presented with physical symptoms of unbalanced gait, a slight left-hand tremor with hypo- and bradykinesia, a loss of smell, and swallowing difficulty in the form of excess saliva accumulation. A life-long athlete and otherwise healthy, she sought medical help as she did not attribute her difficulties to aging. Along with self-reported symptoms, a physical examination identified left-sided bradykinesia and rigidity as well as masklike facial appearance. An MRI ruled out a brain tumor, and a diagnosis of PD was made two months prior to study participation and subsequently confirmed by a second source.
Apart from the PD-associated symptoms, the participant did not disclose additional neurological, motor, communication, or psychological problems. The score of 29/30 on the Montreal Cognitive Assessment screening tool [13] for mild cognitive dysfunction, revealed adequate attention, executive function, memory, language, visuoconstructional skills, conceptual thinking, calculations, and orientation. The answers on the Physical Activity Readiness Questionnaire (PARQ) suggested that the participant had adequate physical ability to partake in an exercise study [14]. For the duration of the study she maintained her current prescribed forms of medication which consisted of a fixed dose of Sinemet to alleviate some of her motor symptoms.

\section{Instrumentation and procedure}

The study was a 17 -week exercise intervention case study $(\mathrm{n}=1)$. The participant completed 23 training sessions over two study phases. Phase 1 consisted of 12 training sessions (Quadmill ${ }^{\mathrm{TM}}$ setting 30 equivalent to 48 platform revolutions per minute (rpm)) completed during a six-week period, with a biweekly session frequency. Phase 2 consisted of 11 additional higher intensity training sessions (Quadmill ${ }^{\mathrm{TM}}$ average setting 38.2 equivalent to 57.7 platform (rpm) completed during an 11-week period, with once-a-week session frequency. Throughout the course of the study, three types of data were collected: biomechanical, physiological, and speech data.

During each of the 23 training sessions the partici-

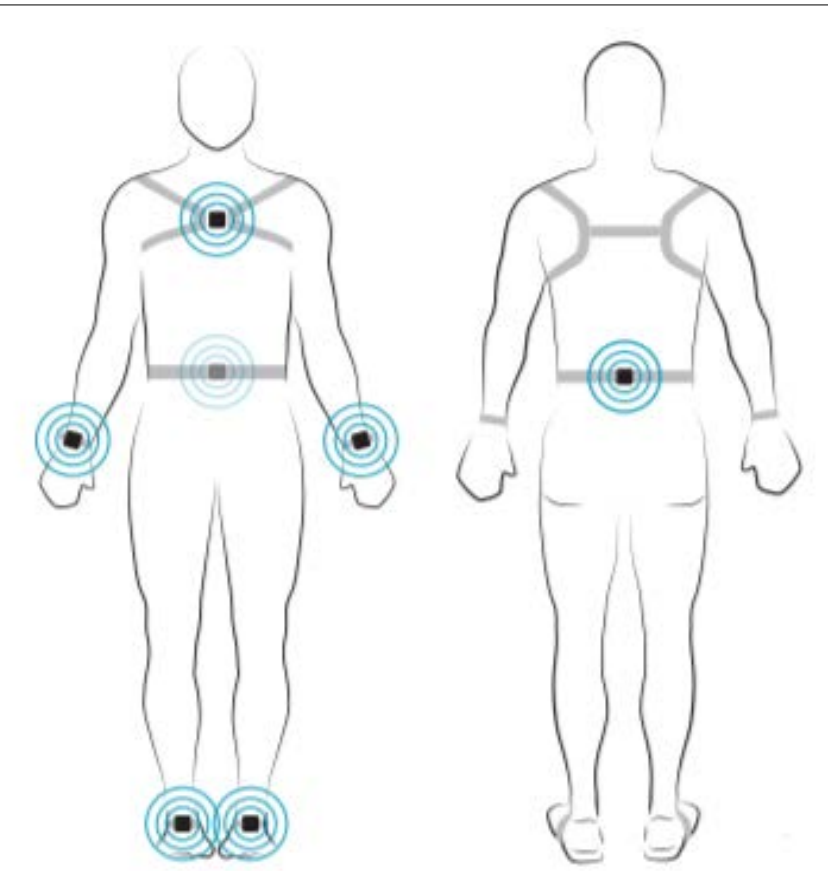

Figure 1: Location of OPAL sensors worn by the participant during "Stand and Walk" (SAW) and static postural stability (SWAY) data collection. 
Citation: Bauer J, Vincent I, Buckenmeyer P, et al. (2018) The Effect of Non-Impact Lower Body Exercise on Individuals with Parkinson's Disease: A Case Study. J Phys Med 1(1):24-31

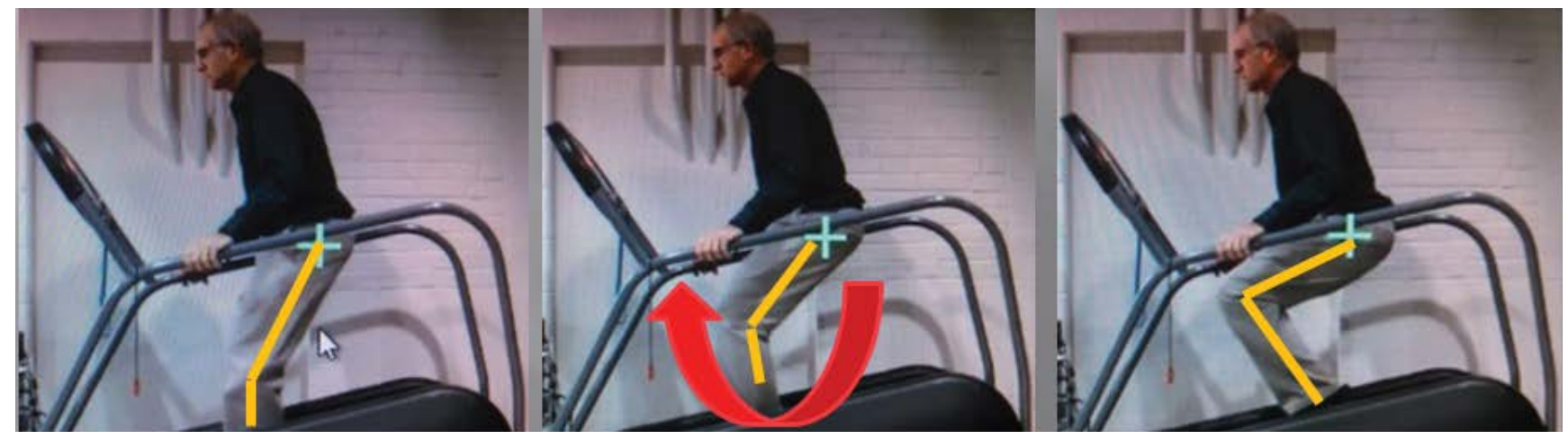

Figure 2: An individual stands upright on a solid platform that moves in an elliptical forward and down, then up and backward path while all motion is absorbed with the lower extremities.

pant began with having resting heart rate (HR) and blood pressure (BP) recorded. Six Mobility Lab ${ }^{\mathrm{TM}}$ OPAL sensors were then fixed on her body to record biomechanical gait and posture data (Figure 1). Pre-exercise data were recorded as she performed a seven-meter stand and walk (SAW) trial and a static postural stability (SWAY) trial. The participant then completed three 45-second bouts of exercise on the Quadmill ${ }^{\mathrm{TM}}$ with two minutes of rest between each exercise bout during which her HR, $\mathrm{BP}$, and rate of perceived exertion (RPE) were recorded by researchers. Post workout SAW and SWAY trials were repeated, and the session was concluded.

\section{Exercise intervention}

Quadmill ${ }^{\mathrm{TM}}$ : The exercise intervention was completed using the Quadmill ${ }^{\mathrm{TM}}$, a non-impact lower body exercise machine manufactured by reACT training systems (Portland, OR). During operation it eccentrically loads the muscles of the lower body as the user maintains an upright motionless posture of the upper body while lightly gripping arm rails for additional support (Figure 2).

The machine was designed to create a low-impact, high-intensity eccentric lower body training experience for the user. Proper technique requires an individual to keep their upper body in a fixed upright trunk position while absorbing all machine generated motion exclusively with their lower body. When in use, the platform follows the motion of two rotating cams that result in the user moving forward/backward and up/down. Total displacement per cycle is $43 \mathrm{~cm}$ in the vertical and horizontal directions. Prior to the start of data collection, the participant was trained to exercise on the Quadmill ${ }^{\mathrm{TM}}$ and it was during that training that the initial machine intensity settings were determined. A control panel allows the user to select from a variety of pre-programmed exercise options. For this study, the manual setting was selected and set at an initial intensity of 30 , which equates to $48 \mathrm{rpm}$. That training level was selected to allow her to comfortably complete three consecutive 45 -second exercise bouts sep- arated with two-minute rest intervals while maintaining RPE scores in the 12-14 range with $\mathrm{HR}<120$ beats/min and peak systolic $\mathrm{BP}<135 \mathrm{mmHg}$.

\section{Biomechanical data collection}

Mobility Lab ${ }^{\mathrm{TM}}$ : Kinematic gait and posture related data were collected both pre-and post-exercise. The data were collected using the Mobility Labs ${ }^{\mathrm{TM}}$ OPALs, which are small wearable inertial measurement units (IMU), each containing a 3-D accelerometer, 3-D gyroscope and 3-D magnetometer capable of collecting data at $250 \mathrm{~Hz}$. The OPALs were secured via Velcro straps and/or adjustable nylon harnesses on the left and right wrists and ankles, the lumbar spine and at the base of the sternum as seen in Figure 1.

Prior to exercise on the Quadmill ${ }^{\mathrm{TM}}$ during each test session, the participant completed two tests that assessed gait and static balance. Data were collected following the APDM Mobility Lab ${ }^{\mathrm{TM}}$ six OPAL SAW 7-m walking test and the SWAY static postural stability protocols $[15,16]$. The SAW test required the subject to first stand as still as possible at a designated area for 30 seconds with her hands on her hips and her eyes open and focused straight ahead. After 30 seconds, the subject was instructed to walk forward at a self-selected pace for seven meters, turn around and return to where she had started. Following completion of the SAW test, the subject performed a SWAY test. The SWAY test required the subject to stand as still as possible with her hands on her hips for 30 seconds with her eyes closed. Immediately post-exercise the SAW and SWAY data collection protocols were repeated. A summary of SAW and SWAY data outcomes are presented in Table 2 and Table 3.

\section{Physiological data collection}

Prior to each exercise session, the participant was seated quietly for five minutes and then had her HR and $\mathrm{BP}$ taken. HR was measured by radial palpation and BP via auscultation and standard cuff. Following each of the 
Citation: Bauer J, Vincent I, Buckenmeyer P, et al. (2018) The Effect of Non-Impact Lower Body Exercise on Individuals with Parkinson's Disease: A Case Study. J Phys Med 1(1):24-31

Table 1: Physiological Responses to Quadmill ${ }^{\mathrm{TM}}$ Exercise for Phase 1 Workload (median [range]).

\begin{tabular}{|l|l|l|l|l|l|l|}
\hline Phase 1 & Workload $(\mathbf{r p m})$ & HR (bpm) & SBP $\mathbf{( m m H g )}$ & DBP $\mathbf{( m m H g )}$ & MAP $(\mathbf{m m H g})$ & RPE \\
\hline Rest & & $61[16]$ & $112[20]$ & $85[14]$ & $94[16]$ & \\
\hline Bout 1 & 48 & $84[40]$ & $121[18]$ & $88[10]$ & $98[12]$ & $14[3]$ \\
\hline Bout 2 & 48 & $89[28]$ & $130[18]$ & $84[10]$ & $100[11]$ & $15[2]$ \\
\hline Bout 3 & 48 & $94[34]$ & $134[20]$ & $84[10]$ & $99[11]$ & $15[2]$ \\
\hline
\end{tabular}

\section{Gait - Lower Limb - Double Support Left} Leg $(\% \mathrm{GCT})$ [mean]

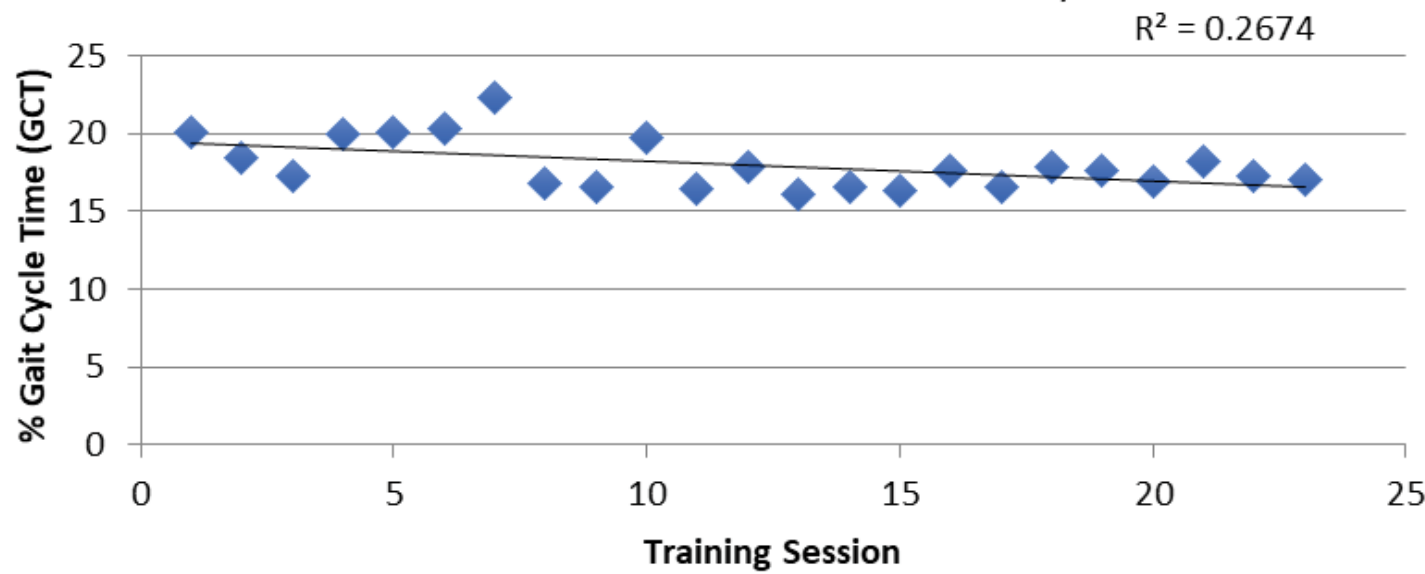

Figure 3: Left foot gait data indicate a trend of decreasing double support as an overall percentage of the gait cycle time.

three 45-second exercise bouts on the Quadmill ${ }^{\mathrm{TM}}$, the participant's HR, BP, and RPE were taken and recorded. A summary of HR, BP, and RPE data outcomes are presented in Table 1 and Table 4.

\section{Speech data}

Speech data were collected six times. During the study, speech samples were obtained at the beginning of training sessions 1, 6, 11, 16, and 22. An additional speech recording session took place seven weeks following completion of the study, at which time the participant was no longer taking Sinemet. Data acquisition took place in a quiet lab space where the participant was comfortably seated in front of a microphone, with a mouth-to-microphone distance of $10 \mathrm{~cm}$. She performed seven tasks at a comfortable pitch and loudness level: a) Three trials for production of the vowel /a/ (such as in "ah") for five seconds; b) Three trials for the longest sustained production of /a/ following a maximum inhalation; c) Three trials for repeating the consonant-vowel syllable /pa/ (such as in "puh") as many times as possible during an eight-second window; d) Three trials for repeating the vowel-vowel combination $/ \mathrm{i}-\mathrm{u} /$ as many times as possible during a six-second window; e) Three productions of the question "Are you leaving today or tomorrow?"; f) Three productions of the sentence "We knew you were away all year."; and g) One reading of a standard reading passage, the Rainbow Passage. The task order was randomized for each speech recording session. The productions were recorded by the Visi-Pitch IV software (Kay Elemetrics, Model 3950), which is designed to record and extract a multitude of motor speech, acoustic, and aerodynamic measures.

\section{Results}

\section{Biomechanical data}

Stability and gait: Graphical analysis of gait and balance data indicated trends of improved function for the participant over her 17-week training. Examples of representative gait and balance data can be seen in Figure 3 and Figure 4.

The measurements recorded for the SAW 7-meter walking task indicated that the exercise bouts on the Quadmill ${ }^{\mathrm{TM}}$ had a positive acute and chronic effect on the participant. Measures related to gait speed, duration, support, and leg swing all indicated changes consistent with return to more controlled and "normal" walking. PD tends to present in slower cadence, increased gait cycle time, an increase in double support, reduced midswing foot elevation, increased stance time, step duration, and decreased stride length. Measurements related to those parameters showed positive directional changes over the course of the study as indicated in the data shown in Table 2. Lower back and trunk values of range of motion also changed over the course of the study and 


\section{Postural Sway - Acc - Mean Velocity (m/s)}

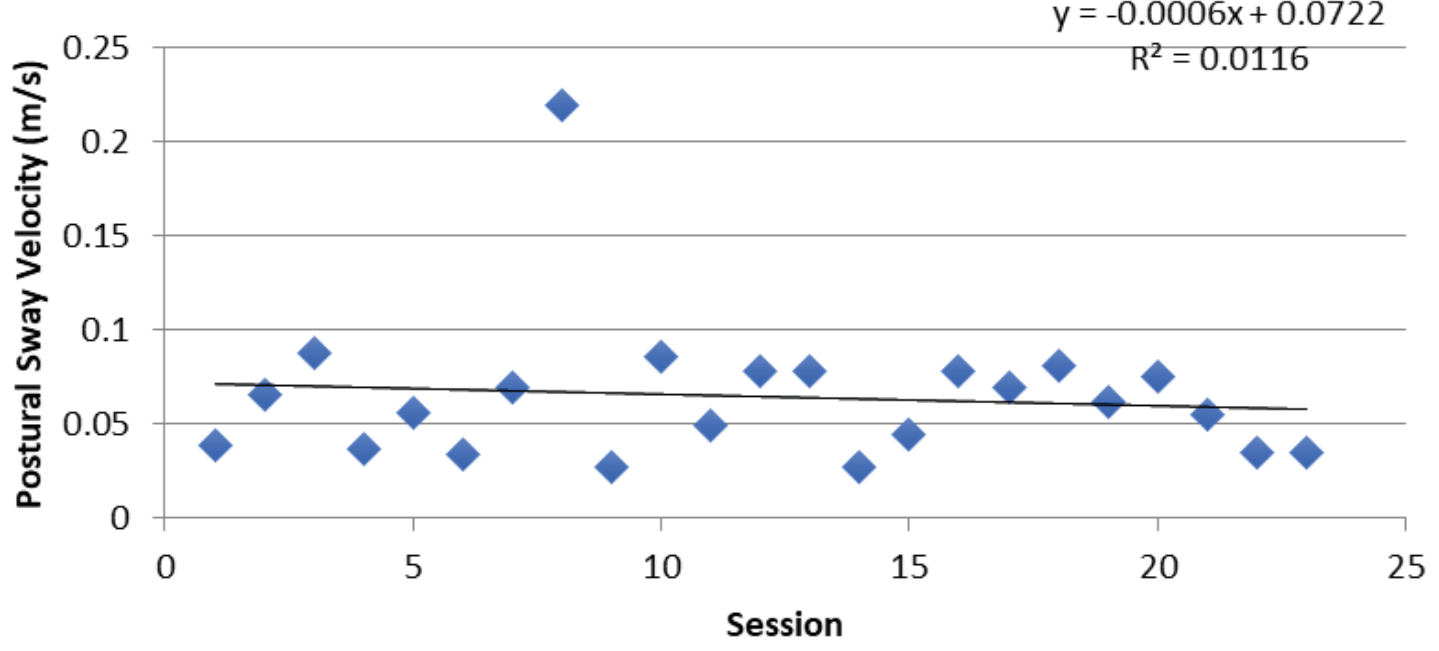

Figure 4: Postural sway velocity indicating a trend of decreased velocity over the course of the training.

Table 2: Gait and Range of Motion during gait changes from session 1 to session 23. Lower Limb Bilateral data shows left and right-side mean values from the last collection date as well as direction of left-and right-side percent changes from start to end of study.

Lower Limb

\begin{tabular}{|c|c|c|c|}
\hline Bilateral & Normative & Session 23 Left | Right & Change from Session 1 Left | Right \\
\hline Cadence (steps/min) & $98.2-129$ & $102 \mid 101$ & $+5.3 \% \mid+4.5 \%$ \\
\hline Gait Cycle Duration (s) & $0.930-1.22$ & $1.18 \mid 1.18$ & $-5.3 \% \mid-4.1 \%$ \\
\hline Gait Speed $(\mathrm{m} / \mathrm{s})$ & $0.990-1.55$ & $1.09 \mid 1.04$ & $+8.6 \% \mid+4.2 \%$ \\
\hline Double Support (\%GCT) & $12.8-25.0$ & $17.9 \mid 18.4$ & $-13.1 \% \mid-10.6 \%$ \\
\hline Elevation at Midswing $(\mathrm{cm})$ & $0.440-2.76$ & $0.590 \mid 0.874$ & $-41.8 \% \mid+57.2 \%$ \\
\hline Stance (\%GCT) & $56.0-62.5$ & $58.7 \mid 59.5$ & $-1.8 \% \mid-2.3 \%$ \\
\hline Step Duration (s) & $0.470-0.620$ & $0.602 \mid 0.582$ & $-5.0 \% \mid-3.6 \%$ \\
\hline Stride Length (m) & $1.04-1.57$ & $1.28 \mid 1.22$ & $+3.2 \% \mid 0.5 \%$ \\
\hline Swing (\%GCT) & $37.5-44.0$ & $41.3 \mid 40.5$ & $+2.7 \% \mid 3.6 \%$ \\
\hline Lumbar Range of Motion & Normative & Session 23 & Change from Session 1 \\
\hline Coronal Range of Motion (deg) & $4.82-13.7$ & 8.73 & $+110.9 \%$ \\
\hline Sagittal Range of Motion (deg) & $3.10-8.27$ & 4.55 & $-30.3 \%$ \\
\hline Transverse Range of Motion (deg) & $5.08-18.1$ & 6.66 & $+27.1 \%$ \\
\hline Trunk Range of Motion & Normative & Session 23 & Change from Session 1 \\
\hline Coronal Range of Motion (deg) & $2.09-8.62$ & 3.60 & $+26.6 \%$ \\
\hline Sagittal Range of Motion (deg) & $3.30-7.60$ & 4.21 & $-5.9 \%$ \\
\hline Transverse Range of Motion (deg) & $4.44-12.6$ & 5.66 & $-0.6 \%$ \\
\hline
\end{tabular}

Table 3: Postural Sway data indicating changes from the beginning to end of study period. Note that the units for the variable Jerk are based on Mancini, Salarian, Carlson-Kuhta, et al. [22].

Postural Sway - Angles

\begin{tabular}{|c|c|c|c|}
\hline Unilateral & Normative & Session 23 & Change from Session 1 \\
\hline Sway Area $\left(\mathrm{deg}^{2}\right)$ & $0.248-1.59$ & 0.275 & $-50.2 \%$ \\
\hline \multicolumn{4}{|c|}{ Postural Sway - Acceleration } \\
\hline Unilateral & Normative & Session 23 & Change from Session 1 \\
\hline Jerk $\left(\mathrm{m}^{2} / \mathrm{s}^{5}\right)$ & $0.516-3.71$ & 0.398 & $-76.5 \%$ \\
\hline Acceleration $\left(\mathrm{m} / \mathrm{s}^{2}\right)$ & $4.20-10.0$ & 3.82 & $-39.6 \%$ \\
\hline
\end{tabular}

were indicative of increased flexibility of the upper body during gait.
Results of the SWAY task indicated that both acute and chronic improvements were made over the course of the study (Table 3). Data collected at the beginning 
Citation: Bauer J, Vincent I, Buckenmeyer P, et al. (2018) The Effect of Non-Impact Lower Body Exercise on Individuals with Parkinson's Disease: A Case Study. J Phys Med 1(1):24-31

Table 4: Physiological Responses to Quadmill ${ }^{\mathrm{TM}}$ Exercise for Phase 2 Workload (median [range]). A comparison of Phase 1 to 2 for HR, SBP, DBP, and MAP did not elicit significant differences despite a 27\% higher workload in Phase 2.

\begin{tabular}{|l|l|l|l|l|l|l|}
\hline Phase 2 & Workload $(\mathbf{r p m})$ & HR $(\mathbf{b p m})$ & SBP $(\mathbf{m m H g})$ & DBP $\mathbf{( m m H g})$ & MAP $(\mathbf{m m H g})$ & RPE \\
\hline Rest & & $64[16]$ & $115[14]$ & $85[30]$ & $95[22]$ & \\
\hline Bout 1 & 57.7 & $86[30]$ & $127[26]$ & $84[28]$ & $98[25]$ & $12[2]$ \\
\hline Bout 2 & 57.7 & $94[32]$ & $128[26]$ & $86[23]$ & $99[24]$ & $13[2]$ \\
\hline Bout 3 & 57.7 & $100[36]$ & $134[18]$ & $85[21]$ & $101[17]$ & $13[3]^{*}$ \\
\hline
\end{tabular}

*RPE was significantly lower in Bout 3 of Phase 2 compared to Phase 1.

Table 5: Maximum phonation time (MPT) of trial participant.

\begin{tabular}{|l|l|l|l|l|l|l|}
\hline $\begin{array}{l}\text { Phase 1: Training } \\
\text { 2x/week }\end{array}$ & & $\begin{array}{l}\text { Phase 2: Training } \\
\text { 1x/week }\end{array}$ & $\begin{array}{l}\text { Seven weeks post- } \\
\text { study follow-up }\end{array}$ & $\begin{array}{l}\text { Normative data } \\
\text { for adult females }\end{array}$ \\
\hline First test & Mid test & Last test & First test & Last test & & \\
\hline $22.3 \mathrm{~s}$ & $19.1 \mathrm{~s}$ & $16.8 \mathrm{~s}$ & $16.6 \mathrm{~s}$ & $17.2 \mathrm{~s}$ & $14.6 \mathrm{~s}$ & $16-35 \mathrm{~s}$ \\
\hline
\end{tabular}

of each test session compared to similar data collected at the end indicated a reduced area of center of pressure migration. The decrease in anterior-posterior and medial-lateral movement of the center of pressure infers better control of static posture with the implied reduction in the likelihood of becoming unstable and falling while standing. Additionally, changes in acceleration and jerk were reduced indicating a slower and more controlled strategy for making changes in balance during quiet standing.

\section{Physiological data}

Exercise data for the 23 training sessions were split into Phase 1 of twelve lower intensity sessions (QuadmillTM setting 30 equivalent to 48 platform $\mathrm{rpm}$ ) and Phase 2 with eleven higher workload intensity sessions (Quadmill $^{\mathrm{TM}}$ average setting 38.2 equivalent to 57.7 platform $\mathrm{rpm}$ ). Data were collected four times (Rest, Bout 1, Bout 2 , Bout 3) during each training session. Rest represents data collected following five minutes of quiet sitting at the start of each training session. Bout 1 , Bout 2 and Bout 3 represent data collected during the two minutes following the three 45-second exercise bouts performed during each training session. A Related-Samples Wilcoxon Signed Rank non-parametric test was utilized to evaluate differences among the HR, BP, and RPE values across exercise Phases 1 and 2. Alpha level for significance was set at the $\mathrm{p}<0.05$ level with the null hypothesis of zero median differences between low and high workout intensity outcomes.

HR (bpm), systolic BP (mmHg), and mean arterial pressure (mmHg) increased (Table 1 and Table 4) from Rest to Bout 3 during training sessions for both Phase 1 and Phase 2. Diastolic BP ( $\mathrm{mmHg}$ ) did not change from Rest to Bout 3 for Phase 1 or Phase 2. RPE for Phase 1 did increase from exercise Bout 1 to Bout 3 (Table 4). Interestingly, RPE was significantly lower during Phase 2 compared to Phase 1. In this case, the RPE (median value [range]) for Bout 3, for Phase 2 (13 [3]) was significantly lower than for Phase 1 (15 [2]).

\section{Speech data}

Motor speech data indicated consistent adequate speech intelligibility, speech sound production precision, and steady voice free of tremor. Voice pitch and loudness variability, as well as speech rate, were consistently mildly reduced. Respiratory support for speech slightly declined as the study progressed, with a noted decline following Sinemet therapy termination following study completion as noted at a seven-week post study follow-up as indicated in Table 5. The decreased maximum phonation time (MPT) noted seven weeks post-study indicated reduced air intake during inhalation compared to previous measurements.

\section{Discussion}

A 17-week training protocol using a Quadmill ${ }^{\mathrm{TM}}$ eccentric lower body exercise machine resulted in measurable improvements in gait and static postural stability for the early-onset PD participant. Physiological variables indicated improved cardio-vascular capability over the period of study. No improvement of speech and voice variables were noted.

A host of biomechanical parameters related to the gait initiation and control as well as static postural stability showed measurable improvement over the course of this training study for the participant. Non-impact lower body exercise using the Quadmill ${ }^{\mathrm{TM}}$ appears to hold promise to reduce some of the most significant physical symptoms of PD when applied to an otherwise healthy newly diagnosed individual. This unique exercise modality required less than 5 minutes of moderate exertion weekly to maintain/improve gait and balance function. Such a small commitment of time and effort should encourage high levels of compliance should it be prescribed for other PD patients.

Several recent investigations have studied the effects of physical activity on PD patients [17-21]. From these studies, there have been consistent findings of improved motor and cognitive function, aerobic capacity, cardi- 
Citation: Bauer J, Vincent I, Buckenmeyer P, et al. (2018) The Effect of Non-Impact Lower Body Exercise on Individuals with Parkinson's Disease: A Case Study. J Phys Med 1(1):24-31

ac sympathetic modulation, skeletal muscle strength, and cardiopulmonary function. An interesting finding from Kanegusuku, et al. [17] study suggests that there is a blunted exercise response in $\mathrm{PD}$ patients regarding cardiopulmonary measures. In particular, $\mathrm{VO}_{2}, \mathrm{HR}$ and systolic BP responses were significantly less than healthy controls at maximal and submaximal exercise efforts. The present study showed a similar type of response in that HR and systolic BP measures were not significantly different when comparing lower workload response with higher workload responses. This finding is consistent with the manifestation of neurodegenerative outcomes associated with PD. In other words, due to a lessened neural and motor function, related sympathetic and parasympathetic responses may become limited in higher intensities of exercise. This can create a "disconnect" with "actual" physical and psychological (subjective) responses during exercise. This was observed in our study wherein the RPE was $10 \%$ less with higher exercise workloads compared to lower exercise workloads. The expected response of RPE with exercise is usually a direct correlation with heart rate, i.e. increased heart rate associated with increased RPE response as workload increases. This may be a result of PD or a relatively "normalizing" response of motor function with regular exercise sessions.

Analysis of speech data did not indicate patient improvement as a result of Quadmill ${ }^{\mathrm{TM}}$ training at the work volume and intensity used in the study. However, it was interesting that MPT was measurably less at seven weeks post-study when compared to data collected during the Phase 1 and 2 exercise sessions. This outcome may have been due to a combination of factors including her cessation of taking Sinemet along with no longer exercising on the Quadmill ${ }^{\mathrm{TM}}$. Further investigation is needed to determine if increased duration and/or intensity of Quadmill ${ }^{\mathrm{TM}}$ training will have beneficial effects regarding speech related PD symptoms.

It is unknown if the improvements we observed in the patient resulted solely from participation in our training study; however, the participant indicated that her medication and daily activities had remained constant over the 17 weeks of data collection. A longitudinal follow-up of the participant has not been conducted so there are no data on whether the observed improvements were maintained for any significant period following the conclusion of the study.

A case study with one individual does not provide enough data to generalize or warrant making broad recommendations regarding its results, but the data collected during this study indicate that training on a Quadmill $^{\mathrm{TM}}$ exercise machine contributed to measurable improvements in many of the most worrisome physical symptoms linked to PD for the participant in the study.
Training on the Quadmill ${ }^{\mathrm{TM}}$ should be studied in more detail with larger segments of the PD population to determine its generalized use to reduce gait deficits and static postural instability resulting from Parkinson's disease.

\section{References}

1. Sanchez J, Hurtado J, de Oliveira A (2017) Stem Cells Transplantation for Treatment of Cognitive Deficits in Parkinson's Disease. Psychology \& Neuroscience 10: 307-313.

2. Shin JY, Pohlig RT, Habermann B (2017) Self-Reported Symptoms of Parkinson's Disease by Sex and Disease Duration. West J Nurs Res 39: 1412-1428.

3. Gröger A, Kolb R, Schäfer R, et al. (2014) Dopamine Reduction in the Substantia Nigra of Parkinson's Disease Patients Confirmed by In Vivo Magnetic Resonance Spectroscopic Imaging. PLoS One 9: e84081.

4. Van Den Eeden SK, Tanner CM, Bernstein AL, et al. (2003) Incidence of Parkinson's disease: variation by age, gender, and race/ethnicity. Am J Epidemiol 157: 1015-1022.

5. Schrag A, Schott J (2006) Epidemiological, clinical, and genetic characteristics of early-onset parkinsonism. Lancet Neurol 5: 355-363.

6. Yokochi M, Narbayashi H (1981) Clinical characteristics of Juvenile Parkinsonism. In: Rose F, Capildeo R, Research Progress in Parkinson's disease. Pitman Medical, London, 35-39.

7. Bhidayasiri R, Reichmann H (2013) Different diagnostic criteria for Parkinson disease: what are the pitfalls? J Neural Transm (Vienna) 120: 619-625.

8. Tysnes O, Storstein A (2017) Epidemiology of Parkinson's Disease. J Neural Transm 124: 901-905.

9. Sujatha J, Rajagopalan S (2017) Visual Changes in Gait in Individuals with Parkinson's Disease. Int J Adv Res Comp Sci 8: 217-220.

10. Saldert C, Bauer M (2017) Multifaceted Communication Problems in Everyday Conversations Involving People with Parkinson's Disease. Brain Science 7: 1-13.

11. Whitfield J, Goberman A (2017) speech Motor Sequence Learning: Effect of Parkinson Disease and Normal Aging on Dual-Task Performance. J Speech Lang Hear Res 60: 1752-1765.

12. Ramig LO, Sapir S, Fox C, et al. (2001) Changes in the vocal loudness following intensive voice treatment (LSVT) in individuals with Parkinson's disease: A comparison with untreated patients and normal age-matched controls. Mov Disord 16: 79-83.

13. Smith T, Gildeh N, Holmes C (2007) The Montreal Cognitive Assessment: validity and utlility in a memory clinic setting. Can J Psychiatry 52: 329-332.

14. Thomas S, Reading J, Shepard RJ (1992) Revision of the Physical Activity Readiness Questionnaire (PAR-Q). Can. J. Spt. Sci. 17: 338-345.

15. Horak FB, Mancini M (2013) Objective biomarkers of balance and gait for Parkinson's disease using body-worn sensors. Mov Disord 28: 1544-1551.

16. Mancini M, Horak FB (2016) Potential of APDM mobility 
Citation: Bauer J, Vincent I, Buckenmeyer P, et al. (2018) The Effect of Non-Impact Lower Body Exercise on Individuals with Parkinson's Disease: A Case Study. J Phys Med 1(1):24-31

lab for the monitoring of the progression of Parkinson's disease. Expert Rev Med Devices 13: 455-462.

17. Duchesne C, Lungu O, Nadeau A, et al. (2015) Enhancing both motor and cognitive functioning in Parkinson's disease: Aerobic exercise as a rehabilitative intervention. Brain Cogn 99: 68-77.

18. Ridgel AL, Walter BL, Tatsuoka C, et al. (2016) Enhanced exercise therapy in Parkinson's Disease: a comparative effectiveness trial. J Sci Med Sport 19: 12-17.

19. Kanegusuku H, Silva-Batista C, Peçanha T, et al. (2016) Blunted maximal and submaximal responses to cardiopulmonary exercise tests in patients with Parkinson Disease
Arch Phys Med Rehabil 97: 720-725.

20. Kanegusuku H, Silva-Batista C, Peçanha T, et al. (2017) Effects of Progressive Resistance Training on Cardiovascular Autonomic Regulation in Patients with Parkinson Disease: A Randomized Controlled Trial. Arch Phys Med Rehabil 98: 2134-2141.

21. Flach A, Jaegers L, Krieger M, et al. (2017) Endurance exercise improves function in individuals with Parkinson's disease: A meta-analysis. Neurosci Lett 659: 115-119.

22. Mancini M, Salarian A, Carlson-Kuhta P, et al. (2012) ISway: a sensitive, valid and reliable measure of postural control. J Neuroeng Rehabil 9: 59. 